\title{
BATS OF CAT TIEN NATIONAL PARK: DIVERSITY, ECHOLOCATION AND TAXONOMIC REMARKS
}

\author{
Vu Dinh Thong \\ Institute of Ecology and Biological Resources, VAST, vietnambat@gmail.com
}

\begin{abstract}
Cat Tien National Park is a hotspot in Vietnam for biodiversity research and conservation. The park, with its extensive areas of primary forest, wetland habitats and large mammal populations, is known worldwide for its outstanding landscapes and biodiversity. Prior to 2007, several bat surveys were carried out in the park. However, data on the park's bats is still limited and the status of previous records of a number of species is unclear. Between 2008 and 2015 , the author conducted a series of bat surveys in the park with emphases on taxonomy and echolocation. Bats were captured using mist nets and four-bank harp traps. Echolocation calls were recorded from inside a flight tent and in natural habitats. Results from the surveys and a literature review indicated that Cat Tien National Park is currently known to be home to 47 bat species belonging to 22 genera and 7 families. Of these, 19 species belonging to 10 genera and 5 families were recorded during field surveys between 2008 and 2015. This paper provides the most recent review of bat species from Cat Tien National Park. Remarks on taxonomy and echolocation frequencies of species complexes are also given in order to assist further research, training, education, management and conservation.
\end{abstract}

Keywords: Chiroptera, conservation, echolocation, Mammalia, training, taxonomy.

\section{INTRODUCTION}

Cat Tien National Park (CTNP) is located in southern Vietnam. With a total area of 73,878 ha, CTNP contains one of the largest remaining natural forests in Vietnam [14]. It "supports a variety of habitat types, including primary and secondary lowland evergreen forest; primary and secondary lowland semi-evergreen forest; freshwater wetlands with open lakes and seasonally inundated grasslands; flooded forest; and a range of secondary habitat types, including grassland and areas" [14]. Prior to the present study, the bat fauna of CTNP had received attention from some scientists and lists of species were included in several publications and grey literature [2]. Of these bat species, several were rarely recorded from other localities in Vietnam and the status of many was unclear. Between 2008 and 2015, the author conducted a series of field surveys in CTNP with an emphasis on bats. Results from the surveys provided new findings with interesting data on taxonomy of bats. This paper presents the most updated review of species diversity and taxonomic remarks of bats from CTNP with recommendation for action plans in the future.

\section{MATERIALS AND METHODS}

\section{Bat capture and identification}

Field surveys were conducted at various habitats of the park, including the sites where specious bat species were recorded in previous surveys. Bats were captured and handled in the field following the guidelines approved by the American Society of Mammalogists [11]. Fourbank harp traps [6] and mist nets of various sizes (2.6 m [height], 3-12 m [length], mesh size: $16 \mathrm{~mm} \times 16 \mathrm{~mm}$ ) were employed to capture bats. Each captured bat was removed carefully from the trap or net and placed individually in a cotton bag. Their external measurements were taken following Bates \& Harrison (1997) [1], Csorba et al. (2003) [5], Vu Dinh Thong (2011) [15] and Vu Dinh Thong et al. $(2012,2012,2012)[19,20,21]$. For taxonomic assessment, external measurements were taken in the field as follows: FA, forearm length: from the extremity of the elbow to the extremity of the carpus with the wings folded; $\mathrm{EH}$, ear height: length of ear conch; TIB, tibia length: from the knee joint to the ankle; HF, hind-foot length: from the extremity of the heel behind the os calcis to the extremity of the 
longest digit, excluding the hairs or claws; Tail, tail length: from the tip of the tail to its base adjacent to the body. Reproductive status and age were assessed following Racey (2009) [9] and Brunet-Rossinni \& Wilkinson (2009) [3], respectively. Some adult males of certain species whose identity could not be confirmed in the field, were collected for taxonomic examination at the Institute of Ecology and Biological Resources. To reduce the influence of seasonal variations in body mass, juveniles and pregnant females were excluded from analyses.

\section{Echolocation recordings and analyses}

Echolocation calls were obtained from recordings in three situations: handheld, inside a flight-tent $(4 \mathrm{~m}$ [length] $\times 4 \mathrm{~m}$ [width] $\times 2 \mathrm{~m}$ [height]) and hand release using a PCTape system at a sampling rate of $480 \mathrm{kHz}$. Batman software, which displays color sonagrams of the detected echolocation signals in real time, was used to obtain high quality sound sequences. Additionally, continuous recordings were also carried out in front of caves and under forest canopies to obtain echolocation calls when bats were leaving their roosts and foraging in natural habitat. All echolocation signals from manual and continuous recordings were analysed using Selena software to measure the constant frequency of the second harmonic (CF2) of each call. The PCTape system, Batman and Selena software are custom-made by the University of Tübingen, Germany.

\section{RESULTS AND DISCUSSION}

\section{Bat diversity of CTNP}

A total of 19 bat species belonging to 10 genera and 5 families were captured during the field surveys between 2008 and 2015 (table 1). Of which, Hipposideros grandis and $H$. cineraceus were commonly recorded (during almost all trapping nights at every study site) while Myotis rosseti appeared as the rarest species with only one individual recorded at a trapping site within the administrative zone of the headquarters of the national park.

Results from the recent surveys and literature review indicate that CTNP is home to a highly diverse bat fauna, which currently comprises 47 species belonging to 22 genera, 7 families (table 2). However, many species, which had been listed from CTNP in the literature, were not recorded during the surveys between 2008 and 2015 .

\section{Taxonomic remarks}

Taxonomic status of Rhinolophus affinis and $R$. pusillus from CTNP is still unconfirmed because their morphological features do not fit well the diagnoses of respective species from other localities in Vietnam. Concise notes and an image of each species are given below.

Rhinolophus affinis: Two individuals of this species were captured from CTNP. The connecting process and sella (fig. 1) of these two individuals compare favourably with Rhinolophus affinis (sensu Csorba et al., 2003). However, their body size and the shape of the anterior nose leaf (horseshoe) differ markedly from Rhinolophus affinis, which is commonly observed in Tam Dao National Park, northern Vietnam and other localities in the country. Echolocation frequency of this unusual form $(83.1-83.7 \mathrm{kHz})$ is distinctly higher than that of the 'typical' Rhinolophus affinis (72.9-73.8 kHz) (Vu Dinh Thong 2011, 2014a, 2014b) [15, 17, 18] (table 3). Extensive studies of the taxonomy, echolocation and genetics of this apparently aberrant population to determine its taxonomic status. In the meantime, it is here treated as Rhinolophus cf. affinis.

Rhinolophus pusillus: Five individual of this species were recorded from the recent surveys. To date, morphological identification is unsatisfactory since pusillus is probably a species complex rather than one discrete taxon [16, 17]. Mean (min-max; sample size) of FA, $\mathrm{EH}, \mathrm{TIB}, \mathrm{HF}$ and Tail of these five individuals are $36.1 \mathrm{~mm}(35.8-36.9 \mathrm{~mm} ; \mathrm{n}=4), 14.8 \mathrm{~mm}$ (13.5-18.0 mm; $\mathrm{n}=5), 14 \mathrm{~mm}(\mathrm{n}=1), 6.2 \mathrm{~mm}$ $(\mathrm{n}=1), \quad 17.5 \mathrm{~mm} \quad(\mathrm{n}=1), \quad$ respectively. Echolocation frequency values for the second harmonic of each calls of the captured individuals is $117.5 \mathrm{kHz}(114.6-119.0 \mathrm{kHz}$; $\mathrm{n}=4$ ). 
Bats of Cat Tien national park

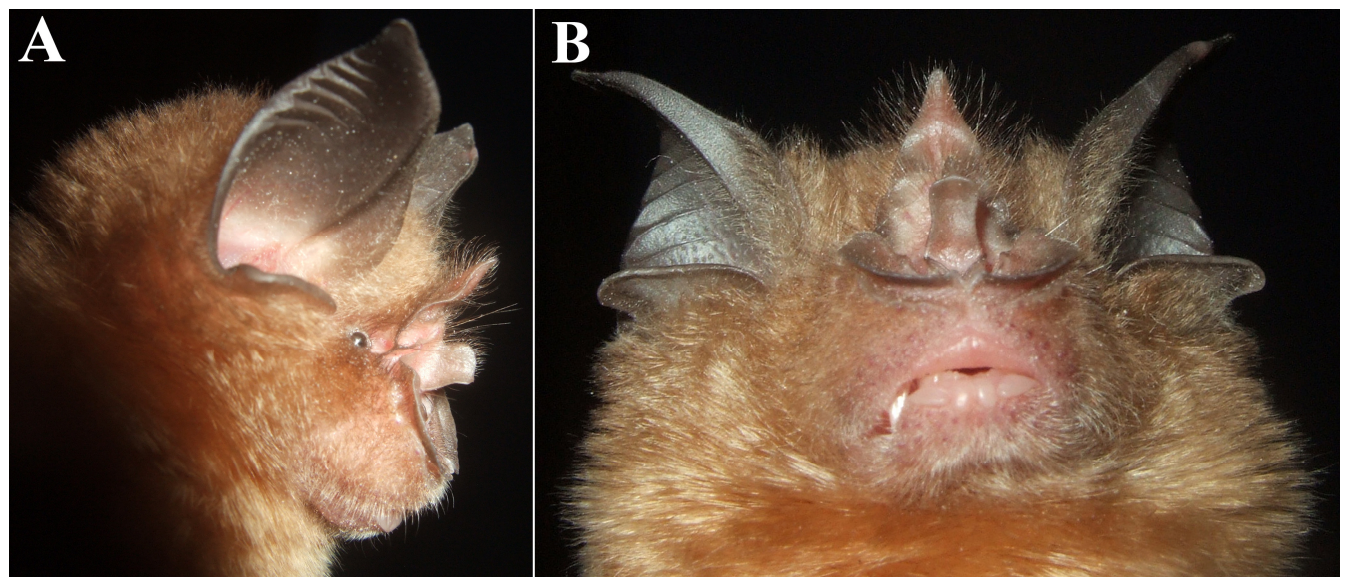

Figure 1. Rhinolophus cf. affinis from CTNP

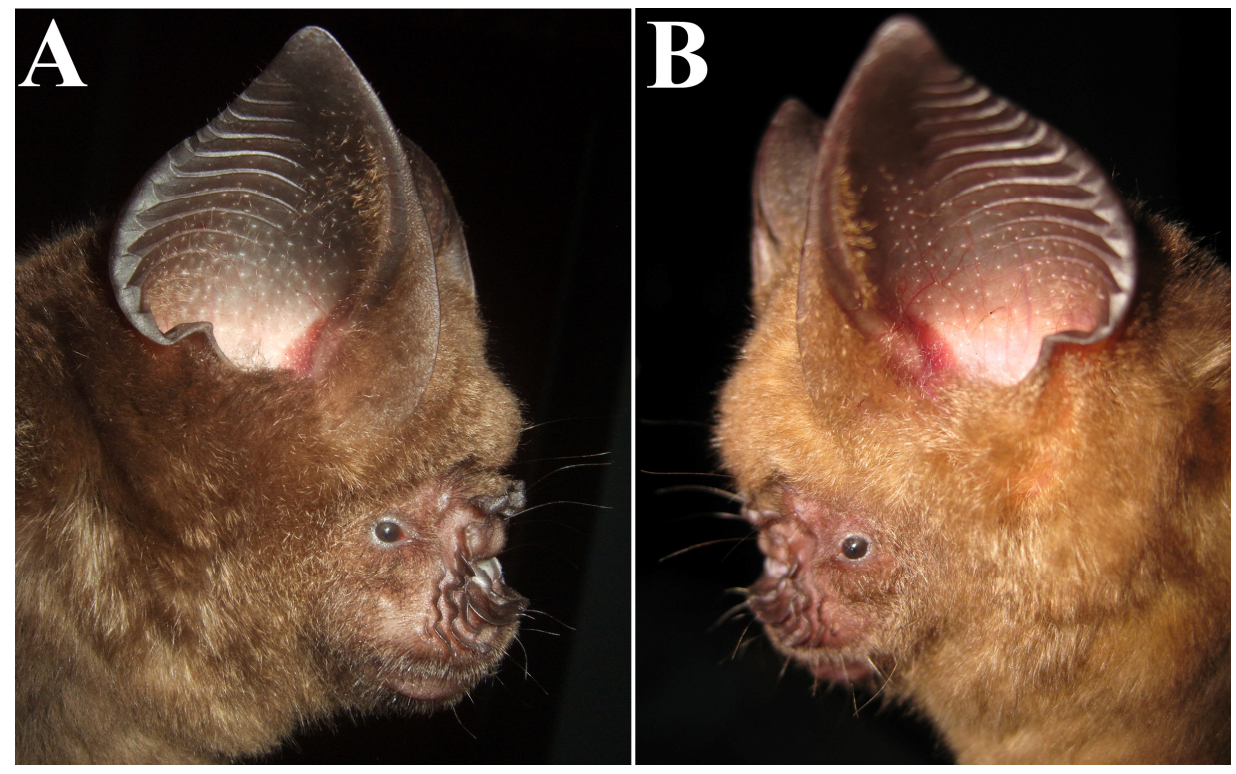

Figure 2. Hipposideros armiger (A) and H. griffini (B)

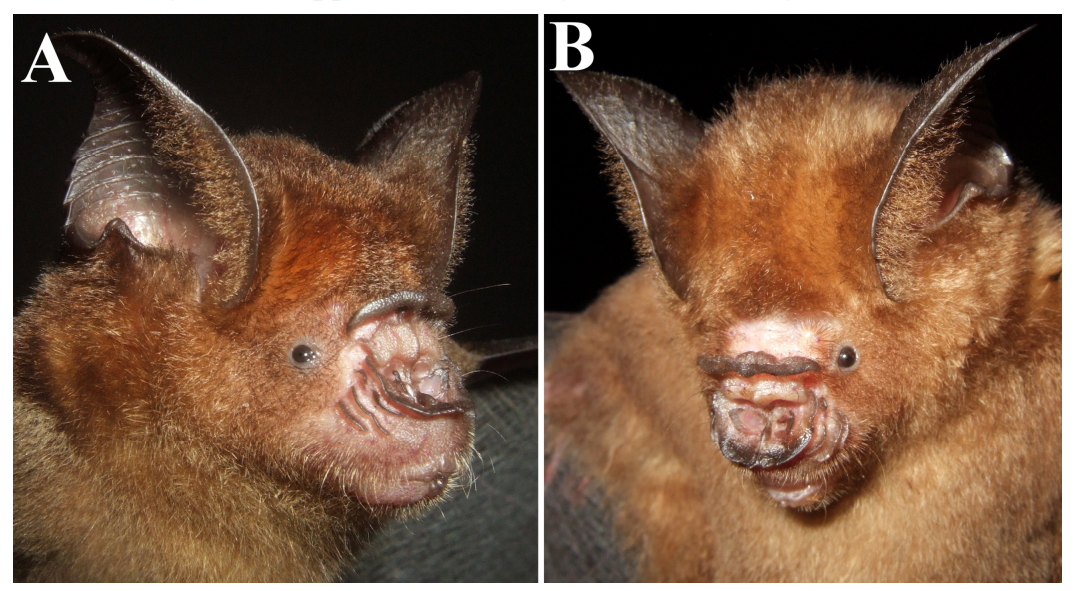

Figure 3. Hipposideros larvatus (A) and H. grandis (B) 
Vu Dinh Thong

Table 1. Bat species recorded from CTNP between 2008 and 2015

\begin{tabular}{|c|c|c|c|c|}
\hline No. & Common name & Latin name & $\begin{array}{c}\text { Conservation } \\
\text { status* }\end{array}$ & $\begin{array}{c}\text { Taxonomic } \\
\text { notes }\end{array}$ \\
\hline & Fruit bats & Pteropodidae & & \\
\hline 1 & Leschenault's Rousette & Rousettus leschenaulti & $\mathrm{LC}$ & Confirmed \\
\hline 2 & Horsfield's short-nosed fruit bat & Cynopterus horsfieldi & LC & Confirmed \\
\hline 3 & Ratanaworabhan's Fruit Bat & Megaerops niphanae & LC & Confirmed \\
\hline 4 & Dawn Bat & Eonycteris spelaea & $\mathrm{LC}$ & Confirmed \\
\hline 5 & $\begin{array}{l}\text { Hill Long-tongued Fruit Bat } \\
\text { False Vampire Bats }\end{array}$ & $\begin{array}{l}\text { Macroglossus sobrinus } \\
\text { Megadermatidae }\end{array}$ & $\mathrm{LC}$ & Confirmed \\
\hline 6 & Lesser False Vampire & Megaderma spasma & $\mathrm{LC}$ & Confirmed \\
\hline 7 & $\begin{array}{l}\text { Greater False Vampire } \\
\text { Old World leaf-nosed bats }\end{array}$ & $\begin{array}{l}\text { Megaderma lyra } \\
\text { Hipposideridae }\end{array}$ & $\mathrm{LC}$ & Confirmed \\
\hline 8 & Griffin’s Leaf-nosed Bat & Hipposideros griffini & N/A & Confirmed \\
\hline 9 & Grand Leaf-nosed Bat & Hipposideros grandis & N/A & Confirmed \\
\hline 10 & Cantor's Leaf-nosed Bat & Hipposideros galeritus & LC & Confirmed \\
\hline 11 & Andersen's Leaf-nosed Bat & Hipposideros pomona & $\mathrm{LC}$ & Confirmed \\
\hline 12 & Least Leaf-nosed Bat & $\begin{array}{l}\text { Hipposideros } \\
\text { cineraceus }\end{array}$ & $\mathrm{LC}$ & Confirmed \\
\hline & Horseshoe bats & Rhinolophidae & & \\
\hline 13 & Indochinese Horseshoe Bat & Rhinolophus chaseni & N/A & Confirmed \\
\hline 14 & Intermediate Horseshoe Bat & Rhinolophus affinis & N/A & Unconfirmed \\
\hline 15 & Lesser Brown Horseshoe Bat & Rhinolophus stheno & LC & Confirmed \\
\hline 16 & Accuminate Horseshoe Bat & $\begin{array}{l}\text { Rhinolophus } \\
\text { acuminatus }\end{array}$ & $\mathrm{LC}$ & Confirmed \\
\hline 17 & $\begin{array}{l}\text { Least Horseshoe Bat } \\
\text { Vesper bats }\end{array}$ & $\begin{array}{l}\text { Rhinolophus pusillus } \\
\text { Vespertilionidae }\end{array}$ & N/A & Unconfirmed \\
\hline 18 & Thick-thumbed Myotis & Myotis rosseti & LC & Confirmed \\
\hline 19 & Lesser Asiatic Yellow House Bat & Scotophilus kuhlii & LC & Confirmed \\
\hline
\end{tabular}

Note: *Conservation status was assessed following the 2015 IUCN Red List of the Threatened Species (http://www.iucnredlist.org/) [13]; N/A=not available; LC=Least Concern.

Hipposideros armiger: No individual of this species was captured during the surveys between 2008 and 2015. Borissenko \& Ivanova (2003) [2] confirmed its occurrence at CTNP. It should be noted that Hipposideros armiger and $H$. griffini are quite similar in external morphology (fig. 2). Detailed information on the taxonomy, echolocation and distribution of H. griffini in Vietnam is given in $\mathrm{Vu}$ Dinh Thong (2012) [16] and Vu Dinh Thong et al. (2012) [20]. These two species are distinguished in echolocation frequency, craniodental characteristics and genetics. An examination of the material of 'Hipposideros armiger' in Borissenko and Ivanova (2003) is required for a taxonomic confirmation. It is likely that both species inhabit CTNP.
Hipposideros larvatus: This was not recorded during the recent surveys between 2008 and 2015. However, Borissenko and Ivanova (2003) included its occurrence at CTNP. To date, Hipposideros larvatus is still treated as a species complex. In fact, this species is indistinguishable from $H$. grandis in morphology (fig. 3) but distinct in genetics (sensu Kruskop, 2013) [8]. $H$. larvatus is smaller than $H$. grandis with a forearm length of 51.5-58.6 mm and 57.6-64.2 $\mathrm{mm}$, respectively (Kruskop 2013). It is remarkable that echolocation frequency of $H$. larvatus $(85-1-95.0 \mathrm{kHz} ; \mathrm{n}=24)$ is lower than that of $H$. grandis $(94.9-104.6 ; \mathrm{n}=109)$ [15]. Within CTNP, echolocation frequency of $H$. grandis is in a range of 94.1-98.2 $(\mathrm{n}=22$; table 3$)$. Further studies in systematics and echolocation of these 
two species are clearly needed in coming time.

Rhinolophus chaseni: This species was originally described from Con Dao National Park of Vietnam by Sanborn (1939) [10]. It was subsequently treated as a either synonym or subspecies of Rhinolophus borneensis [2, 4, 12]. However, Francis (2008) [7] classified it as a distinct species, which was followed by $\mathrm{Vu}$
Dinh Thong (2011), Kruskop (2013) [15, 8]. Without doubt, the material of Rhinolophus borneensis from Vietnam requires an examination for taxonomic confirmation.

Selected morphological measurements and echolocation frequencies of hipposiderids and rhinolophids recorded from CTNP are given in table 3 .

Table 2. An updated species composition of bats from Cat Tien National Park based on the present study results and literature sources

\begin{tabular}{|c|c|c|c|c|}
\hline No. & Common name & Latin name & Source & Taxonomic notes \\
\hline & Fruit bats & Pteropodidae & & \\
\hline 1 & Leschenault's Rousette & Rousettus leschenaulti & $\mathrm{BI}, \mathrm{Kr}, \mathrm{T}$ & Confirmed \\
\hline 2 & Lesser Dog-faced Fruit Bat & Cynopterus brachyotis & $\mathrm{BI}, \mathrm{Kr}$ & Confirmed \\
\hline 3 & Horsfield's short-nosed fruit bat & Cynopterus horsfieldi & $\mathrm{Kr}, \mathrm{T} 14, \mathrm{~T}$ & Confirmed \\
\hline 4 & Greater Shortnosed Fruit Bat & Cynopterus sphinx & $\mathrm{BI}$ & Confirmed \\
\hline 5 & Ratanaworabhan's Fruit Bat & Megaerops niphanae & $\mathrm{BI}, \mathrm{T}$ & Confirmed \\
\hline 6 & Dawn Bat & Eonycteris spelaea & BI, T & Confirmed \\
\hline 7 & $\begin{array}{l}\text { Hill Long-tongued Fruit Bat } \\
\text { Emballonurids }\end{array}$ & $\begin{array}{l}\text { Macroglossus sobrinus } \\
\text { Emballonuridae }\end{array}$ & $\mathrm{BI}, \mathrm{Kr}, \mathrm{T}$ & Confirmed \\
\hline 8 & $\begin{array}{l}\text { Bare-rumped Sheathtail-bat } \\
\text { False Vampire Bats }\end{array}$ & $\begin{array}{l}\text { Saccolaimus saccolaimus } \\
\text { Megadermatidae }\end{array}$ & $\mathrm{BI}$ & Unconfirmed \\
\hline 9 & Lesser False Vampire & Megaderma spasma & $\mathrm{BI}, \mathrm{Kr}, \mathrm{T}$ & Confirmed \\
\hline 10 & $\begin{array}{l}\text { Greater False Vampire } \\
\text { Old World leaf-nosed bats }\end{array}$ & $\begin{array}{l}\text { Megaderma lyra } \\
\text { Hipposideridae }\end{array}$ & $\mathrm{BI}, \mathrm{Kr}, \mathrm{T}$ & Confirmed \\
\hline 11 & Tail-less Leaf-nosed Bat & Coelops frithii & $\mathrm{BI}, \mathrm{Kr}$ & Confirmed \\
\hline 12 & Griffin’s Leaf-nosed Bat & Hipposideros griffini & $\mathrm{T} 12, \mathrm{~T}$ & Confirmed \\
\hline 13 & Great Himalayan Leaf-nosed Bat & Hipposideros armiger & BI & Confirmed \\
\hline 14 & Grand Leaf-nosed Bat & Hipposideros grandis & $\mathrm{T} 11, \mathrm{Kr}, \mathrm{T}$ & Confirmed \\
\hline 15 & Horsfield's Leaf-nosed Bat & Hipposideros larvatus & $\mathrm{BI}$ & Unconfirmed \\
\hline 16 & Cantor's Leaf-nosed Bat & Hipposideros galeritus & $\mathrm{BI}, \mathrm{Kr}, \mathrm{T}$ & Confirmed \\
\hline 17 & Andersen's Leaf-nosed Bat & Hipposideros pomona & $\mathrm{BI}, \mathrm{Kr}, \mathrm{T}$ & Confirmed \\
\hline 18 & $\begin{array}{l}\text { Least Leaf-nosed Bat } \\
\text { Horseshoe bats }\end{array}$ & $\begin{array}{l}\text { Hipposideros cineraceus } \\
\text { Rhinolophidae }\end{array}$ & $\mathrm{BI}, \mathrm{Kr}, \mathrm{T}$ & Confirmed \\
\hline 19 & Indochinese Horseshoe Bat & Rhinolophus chaseni & $\mathrm{T} 11, \mathrm{Kr}, \mathrm{T}$ & Confirmed \\
\hline 20 & Bornean Horseshoe Bat & Rhinolophus borneensis & BI & Unconfirmed \\
\hline 21 & Intermediate Horseshoe Bat & Rhinolphus affinis & $\mathrm{BI}, \mathrm{Kr}, \mathrm{T}$ & Unconfirmed \\
\hline 22 & Lesser Brown Horseshoe Bat & Rhinolophus stheno & $\mathrm{BI}, \mathrm{T}$ & Confirmed \\
\hline 23 & Accuminate Horseshoe Bat & Rhinolophus acuminatus & BI, T & Confirmed \\
\hline 24 & Blyth's Horseshoe Bat & Rhinolophus lepidus & $\mathrm{BI}$ & Unconfirmed \\
\hline 25 & Least Horseshoe Bat & Rhinolophus pusillus & $\mathrm{BI}, \mathrm{Kr}, \mathrm{T}$ & Unconfirmed \\
\hline 26 & $\begin{array}{l}\text { Great Woolly Horsehoe Bat } \\
\text { Vesper bats }\end{array}$ & $\begin{array}{l}\text { Rhinolophus luctus } \\
\text { Vespertilionidae }\end{array}$ & $\mathrm{BI}, \mathrm{Kr}$ & Confirmed \\
\hline 27 & Hardwicke's Woolly Bat & Kerivoula hardwickii & $\mathrm{BI}$ & Confirmed \\
\hline 28 & Papillose Woolly Bat & Kerivoula papillosa & $\mathrm{BI}$ & Confirmed \\
\hline 29 & Himalayan Whiskered Myotis & Myotis siligorensis & $\mathrm{BI}, \mathrm{Kr}$ & Confirmed \\
\hline 30 & Nepalese Whiskered Myotis & Myotis muricola & $\mathrm{BI}, \mathrm{Kr}$ & Confirmed \\
\hline 31 & Peters's Myotis & Myotis ater & $\mathrm{BI}, \mathrm{Kr}$ & Confirmed \\
\hline
\end{tabular}




\begin{tabular}{lllll}
\hline 32 & Thick-thumbed Myotis & Myotis rosseti & $\mathrm{BI}, \mathrm{Kr}, \mathrm{T}$ & Confirmed \\
33 & Myotis & Myotis (Leuconoe) sp. & $\mathrm{BI}$ & Unconfirmed \\
34 & Disk-footed Bat & Eudiscopus denticulus & $\mathrm{BI}$ & Confirmed \\
35 & Common Thick-thumbed Bat & Glischropus tylopus & $\mathrm{BI}$ & Confirmed \\
36 & Kelaart's Pipistrelle & Pipistrellus ceylonicus & $\mathrm{BI}, \mathrm{Kr}$ & Confirmed \\
37 & Javan Pipistrelle & Pipistrellus javanicus & $\mathrm{BI}, \mathrm{Kr}$ & Confirmed \\
38 & Coromandel Pipistrelle & Pipistrellus coromandra & $\mathrm{BI}, \mathrm{Kr}$ & Confirmed \\
39 & Cadorna's pipistrelle & Hypsugo cadornae & $\mathrm{Kr}$ & Confirmed \\
40 & Lesser Bamboo Bat & Tylonycteris pachypus & $\mathrm{BI}, \mathrm{Kr}$ & Confirmed \\
41 & Greater flat-haded Bat & Tylonycteris robustula & $\mathrm{Kr}$ & Confirmed \\
42 & Peters's trumpet-eared Bat & Phoniscus jagorii & $\mathrm{Kr}$ & Confirmed \\
43 & Walston's tube-nosed Bat & Murina walstoni & $\mathrm{Kr}$ & Confirmed \\
44 & Lesser Asiatic Yellow House Bat & Scotophilus kuhlii & $\mathrm{BI}, \mathrm{Kr}, \mathrm{T}$ & Confirmed \\
45 & Blanford's Bat & Hesperoptenus blanfordi & $\mathrm{Kr}$ & Confirmed \\
& Bent-winged Bat & Miniopteridae & & \\
46 & Large Bent-winged Bat & Miniopterus magnater & $\mathrm{BI}, \mathrm{Kr}$ & Confirmed \\
47 & Small Long-fingered Bat & Miniopterus pusillus & $\mathrm{BI}, \mathrm{Kr}$ & Confirmed \\
\hline
\end{tabular}

T11=Vu Dinh Thong (2011) [15]; T12=Vu Dinh Thong (2012) [16]; Kr=Kruskop (2013) [8]; BI=Alex V. Borissenko and Natalia V. Ivanova (unpublished report) [2]; $\mathrm{T}=$ this study.

Table 3. External measurements (in $\mathrm{mm}$ ) and echolocation frequency (in $\mathrm{kHz}$ ) of hipposiderids and rhinolophids recorded from CTNP during this study. Values are given as mean $\pm \mathrm{SD}$, minimummaximum, sample size in parentheses. Abbreviations are defined in the "Material and Methods".

\begin{tabular}{|c|c|c|c|c|c|c|c|}
\hline \multirow{2}{*}{ Species } & \multirow{2}{*}{$\mathrm{n}$} & \multicolumn{5}{|c|}{ Morphological measurements } & \multirow[t]{2}{*}{ Echolocation } \\
\hline & & FA & EH & TIB & HF & Tail & \\
\hline \multirow[t]{2}{*}{ H. cineraceus } & 9 & $34.6 \pm 0.6$ & $15.0 \pm 0.4$ & $14.8 \pm 0.3$ & $4.9 \pm 0.4$ & $25.4 \pm 1.7$ & $153.6 \pm 3.4$ \\
\hline & & $33.8-35.3$ & $14.5-15.5$ & $\begin{array}{c}14.4-15.3 \\
\text { (7) }\end{array}$ & $4.1-5.5(7)$ & $\begin{array}{c}23.0-28.4 \\
\text { (7) }\end{array}$ & $147.8-159.5$ \\
\hline H. galeritus & 2 & $47.0 ; 49.0$ & $\begin{array}{l}14.5 \\
16.0\end{array}$ & $20.1 ; 20.5$ & $6.6 ; 6.7$ & $39.0 ; 43.0$ & $110.1 ; 112.1$ \\
\hline \multirow[t]{2}{*}{ H. grandis } & 22 & $59.8 \pm 1.4$ & $21.3 \pm 0.8$ & - & - & - & $96.3 \pm 1.1$ \\
\hline & & $56.5-62.0$ & $20.0-23.0$ & $\begin{array}{l}21.2 ; 22.1 \\
\text { (2) }\end{array}$ & $\begin{array}{l}9.4 ; 10.0 \\
\text { (2) }\end{array}$ & $\begin{array}{l}33.4 ; 34.3 \\
\text { (2) }\end{array}$ & $94.1-98.2$ \\
\hline \multirow[t]{2}{*}{ H. griffini } & 5 & $85.4 \pm 1.9$ & $29.2 \pm 0.6$ & $36.7 \pm 1.4$ & $15.4 \pm 0.4$ & - & $77.7 \pm 0.9$ \\
\hline & & $83.3-87.2$ & 28.5-30.0 & $35.2-38.7$ & $14.8-15.8$ & - & $76.6-79.2$ \\
\hline \multirow[t]{2}{*}{ H. pomona } & 3 & $41.8 \pm 0.9$ & $22.2 \pm 0.8$ & $18.8 \pm 0.8$ & $5.8 \pm 1.3$ & $33.3 \pm 3.2$ & - \\
\hline & & $40.8-42.6$ & $21.5-23.0$ & 18.4-19.1 & $4.3-6.9$ & $31.0-37.0$ & $122.5 ; 127.9(2)$ \\
\hline $\begin{array}{l}R . \\
\text { accuminatus }\end{array}$ & 1 & 45.87 & 18.18 & 21.39 & 10.04 & 25.43 & 91.4 \\
\hline R. cf affinis & 2 & $48.3 ; 48.5$ & $\begin{array}{l}18.0 \\
19.0\end{array}$ & $21.2 ; 21.5$ & $8.9 ; 9.5$ & $24.2 ; 25.0$ & $83.1 ; 83.5$ \\
\hline \multirow[t]{2}{*}{ R. chaseni } & 3 & $46.5 \pm 0.2$ & $18.3 \pm 1.3$ & $18.4 \pm 0.1$ & $8.0 \pm 0.2$ & $22.2 \pm 2.1$ & $78.4 \pm 1.8$ \\
\hline & & $46.3-46.6$ & $17.0-19.5$ & $18.3-18.5$ & $7.8-8.3$ & $19.8-23.6$ & $76.7-80.2$ \\
\hline \multirow[t]{2}{*}{ R. pusillus } & 5 & $36.1 \pm 0.5$ & $14.8 \pm 1.8$ & - & - & - & $117.5 \pm 1.9$ \\
\hline & & $\begin{array}{c}35.8-36.9 \\
\text { (4) }\end{array}$ & $13.5-18.0$ & $14(1)$ & $6.2(1)$ & $17.5(1)$ & $114.6-119.0(4)$ \\
\hline \multirow[t]{2}{*}{ R. stheno } & 3 & $47.0 \pm 0.4$ & $18.5 \pm 0.5$ & $20.5 \pm 0.4$ & $8.4 \pm 0.2$ & $20.9 \pm 1.1$ & $87.0 \pm 0.2$ \\
\hline & & $46.7-47.5$ & $18.0-19.0$ & $20.2-20.9$ & $8.2-8.6$ & $19.6-21.6$ & $86.8-87.1$ \\
\hline
\end{tabular}




\section{CONCLUSION}

Cat Tien National Park is currently known as a home to 47 species, 22 genera, and 7 families. Of these, 7 species are unconfirmed and at least 5 'species' probably comprise species complexes: Rhinolophus affinis, $R$. pusillus, Hipposideros galeritus, H. larvatus and $H$. pomona. Echolocation frequencies and morphological parameters of these five 'species' from CTNP differ considerably from other populations in Vietnam and worldwide.

Acknowledgements: This research is funded by the Vietnam National Foundation for Science and Technology Development (NAFOSTED) under grant number 106.11-2012.02. The author is very grateful to Prof. Hans-Ulrich Schnitzler, Dr. Annette Denzinger, Dr. Christian Dietz of the University of Tübingen, Germany; Dr Paul J. J. Bates and the late Dr. David L. Harrison of the Harrison Institute, England; Prof. Paul Racey of the University of Exeter, England; Dr. Neil Furey of the Fauna and Flora International (Cambodia); Dr. Tigga Kingston of the Texas Tech University (USA); and to our colleagues at IEBR, CTNP and other institutions for their help and support.

\section{REFERENCES}

1. Bates P. J. J., Harrison D. L., 1997. Bats of the Indian Subcontinent. Harrison Zoological Museum, Sevenoaks, Kent, United Kingdom, 297 pp.

2. Borissenko A. V., Ivanova N. V., 2003. Ecological studies of bats (Mammalia: Chiroptera) in Cat Tien National Park [unpublished report]: $45 \mathrm{pp}$.

3. Brunet-Rossinni A. K., Wilkinson G. S., 2009. Methods for age estimation and the study of senescence in bats. In: Kunz T. H., Parsons S. (eds.) Ecological and Behavioral Methods for the Study of Bats, 2nd edition, Johns Hopkins University Press, Baltimore, pp. 315-325.

4. Corbet G. B., Hill J. E., 1992. The Mammals of the Indomalayan Region. Oxford University Press, Oxford, England, 496 pp.

5. Csorba G., Ujhelyi P., Thomas N., 2003.
Horseshoe Bats of the World (Chiroptera: Rhinolophidae). Alana Books, England, 160 pp.

6. Francis C. M., 1989. A comparison of mist nets and two types of harp traps for capturing bats. Journal of Mammalogy, 70: 865-870.

7. Francis C. M., 2008. A guide to the Mammals of Southeast Asia. Princeton University Press, Princeton, USA, 392 pp.

8. Kruskop S. V., 2013. Bats of Vietnam: Checklist and an identification manual. Moscow, Russia, 299 pp.

9. Racey P. A., 2009. Reproductive assessment in bats. In: Kunz T.H., Parsons S. (eds.) Ecological and Behavioral Methods for the Study of Bats, 2nd edition, Johns Hopkins University Press, Baltimore, pp. 249-264.

10. Sanborn C. C., 1939. Eight new bats of the genus Rhinolophus. Zoological series of Field Museum of Natural History, 24(5): 20 pp.

11. Sikes R. S., W. L. Gannon, and the Animal Care and Use Committee of the American Society of Mammalogists, 2011. Guidelines of the American Society of Mammalogists for the use of wild mammals in research. Journal of Mammalogy, 92: 235-253.

12. Simmons N. B., 2005. Order Chiroptera. In: Wilson DE and Reeder DM (eds.) Mammal species of the world: A taxonomic and geographic reference. The Johns Hopkins University Press, Baltimore, 3rd edition, pp. 312-529.

13. The IUCN Red List of Threatened Species. Version 2015-3. <www.iucnredlist.org>. Downloaded on 11 November 2015.

14. Tordoff A. W., Tran Q. B., Nguyen T. D., Le M. H. 2004. Source Book of Existing and Proposed Protected Areas in Vietnam, Birdlife International Indochina, Hanoi.

15. Vu Dinh Thong, 2011. Systematics and echolocation of rhinolophoid bats (Mammalia: Chiroptera) in Vietnam. PhD Thesis, University of Tuebingen, Tuebingen, Germany, 258 pp. 
16. $\mathrm{Vu}$ Dinh Thong, 2012. New records of Griffin's Leaf-nosed bat (Hipposideros griffini Thong et al. 2012) from Vietnam. Tap chi Sinh hoc, 34(3): 323-327.

17. Vu Dinh Thong, 2014. Acoustic identification and taxonomic remarks of horseshoe bats (Chiroptera: Rhinolophidae) in Cat Ba National Park, northeastern Vietnam. Proceedings of the first VASTBAS workshop on science and technology, Ha Long city, Vietnam: 323-328.

18. Vu Dinh Thong, 2014. Acoustic identification and taxonomic remarks of hipposiderids and rhinolophids (Chiroptera: Hipposideridae, rhinolophidae) in Tam Dao National Park, northeastern Vietnam. Tap chi Sinh hoc, 36(4): 487-493.

19. Vu Dinh Thong, Dietz C., Denzinger A.,
Bates P. J. J., Puechmaille S. J., Callou C., Schnitzler H.-U., 2012. Resolving a mammal mystery: the identity of Paracoelops megalotis (Chiroptera: Hipposideridae). Zootaxa, 3505, 75-85.

20. $\mathrm{Vu}$ Dinh Thong, Puechmaille S. J., Denzinger A., Bates P. J. J., Dietz C., Csorba G., Soisook P., Teeling E. C., Matsumura S., Furey N., Schnitzler H.U., 2012. Systematics of the Hipposideros turpis complex and a description of a new subspecies from Vietnam. Mammal Rev., 42: 166-192.

21. Vu Dinh Thong, Puechmaille S. J., Denzinger A., Dietz C., Csorba G., Bates P. J. J., Teeling E. C., Schnitzler H. U., 2012. A new species of Hipposideros (Chiroptera: Hipposideridae) from Vietnam. Journal of Mammalogy, 93: 1-11.

\section{DƠI CỦA VƯờn QUỐC GIA CÁT TIÊN: TÍNH ĐA DẠNG, TIẾNG KÊU SIÊU ÂM VÀ NHỮNG NHẬN ĐỊNH VỀ PHÂN LOẠI HỌC}

\section{Vũ Đình Thống}

Viện Sinh thái và Tài nguyên sinh vật, Viện Hàn lâm KH \& CN Việt Nam

\section{TÓM TẮT}

Vườn Quốc gia Cát Tiên là một điểm nóng của Việt Nam đối với công tác nghiên cứu và bảo tồn đa dạng sinh học. Vườn quốc gia này cũng nổi tiếng trên thể giới bởi vẻ đẹp nổi bật của cảnh quan và giá trị đa dạng sinh học với sự tồn tại diện tích rừng nguyên sinh rộng, sinh cảnh đất ngập nước và những quần thể thú lớn. Trước năm 2007, đã có một số đợt điều tra về dơi ở vườn quốc gia này. Tuy nhiên, những dẫn liệu về dơi ở đây còn rất hạn chế và nhiều ghi nhận trước đây chưa chắc chắn. Từ 2008 đến 2015 , tác giả đã điều tra về dơi ở vườn quốc gia này với trọng tâm nghiên cứu về phân loại học và tiếng kêu siêu âm. Dơi được bắt bằng bẫy thụ cầm loại bốn khung dây và lưới mờ. Tiếng kêu siêu âm được ghi trong những tình huống khác nhau, bao gồm những tình huống dơi ở trong màn bay và khi dơi kiếm ăn trong sinh cảnh sống tự nhiên của chúng. Kết quả điều tra và tổng hợp từ những tài liệu đã công bố trước đây cho thấy Vườn Quốc gia Cát Tiên là nơi sinh sống của 47 loài dơi thuộc 22 giống, 7 họ. Trong đó, 19 loài thuộc 10 giống, 5 họ được ghi nhận qua các đợt điều tra thực địa từ năm 2008 đến 2015. Bài báo này cung cấp dẫn liệu cập nhật nhất về thành phần loài dơi ở Vườn Quốc gia Cát Tiên. Thêm vào đó, những nhận định về phân loại học và tần số tiếng kêu siêu âm của những tổ hợp loài cũng được bàn luận làm cơ sở cho công tác nghiên cứu, đào tạo, giáo dục, quản lý và bào tồn.

Tù khóa: Chiroptera, bảo tồn, tiếng kêu siêu âm, Mammalia, đào tạo, phân loại học.

Ngày nhận bài: 15-6-2015 